International Journal of Pure and Applied Mathematics

Volume 115 No. 4 2017, 665-672

ISSN: 1311-8080 (printed version); ISSN: 1314-3395 (on-line version)

url: http://www.ijpam.eu

doi: $10.12732 /$ ijpam.v115i4.2

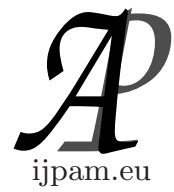

\title{
ASYMPTOTIC DERIVATION OF Z* STATISTIC
}

\author{
Tareq A.M. Atiany ${ }^{1}$, Shamshuritawati Sharif ${ }^{2}$ \\ ${ }^{1,2}$ School of Quantitative Sciences \\ Universiti Utara Malaysia \\ 06010 UUM Sintok, Kedah, MALAYSIA
}

\begin{abstract}
In multivariate analysis, the stability of correlation matrix is a major issue. We can be seen it in the literature, the most popular tests and widely used are Jennrich test and Box M test which introduced by Jennrich in 1970 and Box in 1949. Jennrich test involve inverse of matrix and Box involve the determinant of the matrix. Under these conditions the computation of the tests are quite cumbersome when the data in high dimension. This encourage us to propose a new statistical test. Which is constructed based on upper-offdiagonal elements to overcome the difficulty.
\end{abstract}

Key Words: correlation structure, vector operator, linear transformation

\section{Introduction}

The problem of testing the stability or homogeneity of the correlation matrix is a serious problem. It has been implemented by several researchers e.g. [4], [9], [17], [12], [11], [18], [5].

To test the stability of correlation or covariance matrix the most and widely tests used are [2] and [8] as a example see [4], [12], [11], [18], [6], [5]. These tests apply to several of independent samples of correlation matrices, which are drawn from a $p$ - variate normal distribution. These tests constructed based on likelihood ratio test (LRT) where their distributions under $H_{0}$ were derived for asymptotic case. Moreover, those tests are to test the stability of the correlation matrix in simultaneous way.

$\begin{array}{lr}\text { Received: } & \text { July 25, } 2016 \\ \text { Revised: } & \text { May 2, } 2017 \\ \text { Published: } & \text { August 1, } 2017 \\ \text { \$Correspondence author }\end{array}$

(c) 2017 Academic Publications, Ltd. url: www.acadpubl.eu 
However, usually the number of variables are large. This condition can make the computation of those tests tedious since those tests involves determinant and inverse of the matrix that makes the computation efficiency becomes low $[7]$.

We constructed our test based on vec operator and commutation matrix. This statistical test is based on the linear transformation that change the matrix to vector where it is elements are the upper-off-diagonal only to ensure the nonsingularity of the matrix.

\section{Asymptotic Derivation}

To construct the statistical test we use the asymptotic distribution of the correlation matrix developed in [3] and [14]. Let $X_{1}, X_{2}, \ldots X_{n}$ a random sample of size $n$ drawn from $p$ - variate normal distribution with positive definite covariance matrix $\Sigma$,

$$
S=\frac{1}{n-1} \sum_{i=1}^{n}\left(X_{i}-\bar{X}\right)\left(X_{i}-\bar{X}\right)^{t}
$$

is the sample covariance matrix, where $\bar{X}=\frac{1}{n} \sum_{i=1}^{n}\left(X_{i}\right)$.

The sample correlation matrix $R=S_{d}^{-1 / 2} S S_{d}^{-1 / 2}$ and the population correlation matrix is $\Omega=\Sigma_{d}^{-1 / 2} \Sigma \Sigma_{d}^{-1 / 2}$ where $S_{d}$ and $\Sigma_{d}$ is the diagonal elements of $S$ and $\Sigma$ respectively. Thus the correlation matrix $R$ and $\Omega$ are

$$
\begin{aligned}
R= & {\left[\begin{array}{cccc}
\frac{1}{\sqrt{s_{11}}} & 0 & \ldots & 0 \\
0 & \frac{1}{\sqrt{s_{22}}} & \cdots & 0 \\
\vdots & \vdots & \ddots & \vdots \\
0 & 0 & \cdots & \frac{1}{\sqrt{s_{p p}}}
\end{array}\right]\left[\begin{array}{cccc}
s_{11} & s_{12} & \ldots & s_{1 p} \\
s_{21} & s_{22} & \ldots & s_{2 p} \\
\vdots & \vdots & \ddots & \vdots \\
s_{p 1} & s_{p 2} & \cdots & s_{p p}
\end{array}\right] } \\
& \times\left[\begin{array}{cccc}
\frac{1}{\sqrt{s_{11}}} & 0 & \cdots & 0 \\
0 & \frac{1}{\sqrt{s_{22}}} & \cdots & 0 \\
\vdots & \vdots & \ddots & \vdots \\
0 & 0 & \cdots & \frac{1}{\sqrt{s_{p p}}}
\end{array}\right],
\end{aligned}
$$




$$
\begin{aligned}
\Omega & =\left[\begin{array}{cccc}
\frac{1}{\sqrt{\Omega_{11}}} & 0 & \cdots & 0 \\
0 & \frac{1}{\sqrt{\Omega_{22}}} & \cdots & 0 \\
\vdots & \vdots & \ddots & \vdots \\
0 & 0 & \cdots & \frac{1}{\sqrt{\Omega_{p p}}}
\end{array}\right]\left[\begin{array}{cccc}
\Omega_{11} & \Omega_{12} & \ldots & \Omega_{1 p} \\
\Omega_{21} & \Omega_{22} & \ldots & \Omega_{2 p} \\
\vdots & \vdots & \ddots & \vdots \\
\Omega_{p 1} & \Omega_{p 2} & \cdots & \Omega_{p p}
\end{array}\right] \\
& \times\left[\begin{array}{cccc}
\frac{1}{\sqrt{\Omega_{11}}} & 0 & \ldots & 0 \\
0 & \frac{1}{\sqrt{\Omega_{22}}} & \cdots & 0 \\
\vdots & \vdots & \ddots & \vdots \\
0 & 0 & \cdots & \frac{1}{\sqrt{\Omega_{p p}}}
\end{array}\right] .
\end{aligned}
$$

Then

$$
\rho_{i j}=\frac{\sigma_{i j}}{\sqrt{\sigma_{i i}} \sqrt{\sigma_{j j}}} \text { and } r_{i j}=\frac{s_{i j}}{\sqrt{s_{i i}} \sqrt{s_{j j}}}
$$

\section{Asymptotic Distribution of $v e c(R)$}

The asymptotic sample correlation matrix $R$ will be examined from $p=2$ to $p>2$. For that purpose we use the results presented in [1], see p. 132, Theorem 4.2.3.

Theorem 1. Let $U(n)$ be a sequence of p-component random vectors and $b$ a fixed vector such that $\sqrt{n}[U(n)-b] \stackrel{d}{\rightarrow} N(0, \gamma)$ as $n \rightarrow \infty$. Let $f(u)$ be a vector valued function of $u$ such that each component $f_{j}(u)$ satisfies $\frac{\partial f_{j}(u)}{\partial u_{i}}{ }_{u=b} \neq$ 0 . If ${\frac{\partial f_{j}(u)}{\partial u_{i}}}_{u=b}$ is the $(i, j)^{\text {th }}$ component of $\omega$.Then $\sqrt{n}[f(U(n))-f(b)] \stackrel{d}{\rightarrow}$ $N\left(0, \omega^{t} \gamma \omega\right)$.

For $p=2$ the correlation matrix $\left(\begin{array}{cc}1 & r_{12} \\ r_{21} & 1\end{array}\right)$ this matrix is transforms into vector which is element is $r_{12}$ the upper-off-diagonal of the matrix. This transformation is developed in [16] and we developed it here. However, before we start we need to identify the distribution of correlation sample $R$. By using the vec operator and the commutation matrix, it is formulated in the following proposition. 
Proposition 1. Let $X_{1}, X_{2},, X_{n}$ be a random vector drawn from $p$-varite normal distribution of size $n$ then $\sqrt{n-1}(\operatorname{vec}(R)-\operatorname{vec}(\Omega)) \stackrel{d}{\rightarrow} N(0, \Gamma)$. Here:

i. $\Gamma=2 N_{p} \phi N_{p}$.

ii. $N_{p}=\frac{1}{2}\left(I_{p^{2}}+K p p\right)$.

iii. $\phi=\left(I_{p^{2}}-\left(I_{p} \otimes \Omega\right) \Lambda_{p}\right)(\Omega \otimes \Omega)\left(I_{p^{2}}-\Lambda_{p}\left(I_{p} \otimes \Omega\right)\right)$.

iv. $K_{p p}=\sum_{i=1}^{p} \Sigma_{i=1}^{p}\left(G_{i j} \otimes G_{i j}^{t}\right)$ is the commutation matrix of size $\left(p^{2} \times p^{2}\right)$.

v. $\Lambda_{p}=\Sigma_{i=1}^{p} h_{i} h_{i}^{t} \otimes h_{i} h_{i}^{t}$ where, $h_{i}$ is the $i$-th column of $I_{p}$.

According to Central Limit Theorem, we have the following proposition of covariance matrix of $\operatorname{vec}(S)$.

Proposition 2. If $n \rightarrow \infty$, according to central limit Theorem, the asymptotic distribution of $S$ is equal to $\sqrt{n-1}(\operatorname{vec}(S)-\operatorname{vec}(\Sigma)) \stackrel{d}{\rightarrow} N(0, \operatorname{var}(S))$. Where the covariance of $\operatorname{vec}(S)=\left(I_{p^{2}}+K_{p p}\right)(\Sigma \otimes \Sigma)$.

\section{Asymptotic Distribution of $v\left(R_{U}\right)$}

The correlation matrix is a symmetric matrix, it has redundant elements [15]. To eliminate those elements, we consider the upper off diagonal elements in the matrix only. We denote it $v\left(R_{U}\right)$. For that we present linear transformation matrix $\mathrm{T}$ to eliminate the non-random elements in $R$.

$$
T_{i, j}^{a}= \begin{cases}1,(i, j)=\left(\left(C_{2}^{a}\right)-a+b+1, b\right) & \text { for } b=1,2, \ldots, a-1, \\ 0, & \text { otherwise }\end{cases}
$$

The transformation $T$ presented in matrix form as a block matrix $T=$ $\left(T_{1}\left|T_{2}\right| \ldots \mid T_{p}\right)$ of size $\left(k \times p^{2}\right)$ partitioned into $p$ blocks where $k=\frac{p(p-1)}{2} ; T_{a}=$ $\left(t_{i, j}^{a}\right)$, each of size $(k \times p)$, where $T_{1}$ is zero matrix, where $a=2,3,, p$. Where $C_{2}^{a}$ is the number of combinations of 2 out of a objects.

We use this asymptotic distribution of $R$ to drive the asymptotic distribution of our test.

Proposition 3. Let $X_{1}, X_{2}, \ldots, X_{n}$ is a random sample of size $n$ from . If $\Omega$ is correlation matrix then, $\sqrt{n-1}\left(\left\|v\left(R_{U}\right)\right\|^{2}-\left\|v\left(\Omega_{U}\right)\right\|^{2}\right) \stackrel{d}{\rightarrow} N\left(0, \sigma^{2}\right)$, $\sigma^{2}=4\left(v\left(\Omega_{U}\right)\right)^{t} * T * \Gamma * T^{t} * v\left(\Omega_{U}\right)=8 *\left(v\left(\Omega_{U}\right)\right)^{t} * T * N_{p} * \phi * N_{p} * T^{t} * v\left(\Omega_{U}\right)$.

Proof. The right side $\left(v\left(\Omega_{U}\right)\right)^{t} * T * N_{p} * \phi * N_{p} * T^{t} * v\left(\Omega_{U}\right)$, where $\Gamma=2 N_{p} \phi N_{p}, N_{p}=\frac{1}{2}\left(I_{p^{2}}+K p p\right)$ and $K_{p p}=\Sigma_{i=1}^{p} \Sigma_{i=1}^{p}\left(G_{i j} \otimes G_{i j}^{t}\right)$ is the commutation matrix of size $\left(p^{2} \times p^{2}\right) . \phi=\left(I_{p^{2}}-\left(I_{p} \otimes \Omega\right) \Lambda_{p}\right)(\Omega \otimes \Omega)\left(I_{p^{2}}-\Lambda_{p}\left(I_{p} \otimes \Omega\right)\right)$, 
$\Lambda_{p}=\Sigma_{i=1}^{p} h_{i} h_{i}^{t} \otimes h_{i} h_{i}^{t}$ where, $h_{i}$ is the i-th column of $I_{p}$.

$\left(v\left(\Omega_{U}\right)\right)^{t} * T * N_{p} * \phi * N_{p} * T^{t} * v\left(\Omega_{U}\right)=\left(v\left(\Omega_{U}\right)\right)^{t} * T * \frac{1}{2}\left(I_{p^{2}}+K p p\right) *$ $\left(I_{p^{2}}-\left(I_{p} \otimes \Omega\right) \Lambda_{p}\right)(\Omega \otimes \Omega)\left(I_{p^{2}}-\Lambda_{p}\left(I_{p} \otimes \Omega\right)\right) * \frac{1}{2}\left(I_{p^{2}}+K p p\right) * T^{t} * v\left(\Omega_{U}\right)$

$=\frac{1}{4} *\left[\left(v\left(\Omega_{U}\right)\right)^{t} * T * I_{p}^{2}+\left(v\left(\Omega_{U}\right)\right)^{t} * T * K_{p p}\right]\left[\left(I_{p^{2}}-\left(I_{p} \otimes \Omega\right) \Lambda_{p}\right)(\Omega \otimes \Omega)\right.$

$\left.\left(I_{p^{2}}-\Lambda_{p}\left(I_{p} \otimes \Omega\right)\right)\right] *\left[I_{p}^{2} * T^{t} * v\left(\Omega_{U}\right)+K_{p p} * T^{t} * v\left(\Omega_{U}\right)\right]$.

Since $T * I_{p}^{2}=T, K_{p p} \operatorname{vec}(\Omega)=\operatorname{vec}(\Omega)$ and $(\operatorname{vec}(\Omega))^{t} * K_{p p}=(\operatorname{vec}(\Omega))^{t}$.

Then

$=\frac{1}{4} *\left[\left(v\left(\Omega_{U}\right)\right)^{t} * T+\left(v\left(\Omega_{U}\right)\right)^{t} * T * K_{p p}\right]\left[\left(I_{p^{2}}-\left(I_{p} \otimes \Omega\right) \Lambda_{p}\right)\right](\Omega \otimes \Omega)$

$\left[\left(I_{p^{2}}-\Lambda_{p} *\left(I_{p} \otimes \Omega\right)\right)\right]\left[I_{p}^{2} * T^{t} * v\left(\Omega_{U}\right)+K_{p p} * T^{t} * v\left(\Omega_{U}\right)\right]$.

Corollary 1. Let $\lambda$ a matrix of size $p \times p$ such that $\operatorname{vec}(\lambda)=T^{t} * T * v e c(\Omega)$. Then by using the corollary 1 we have the following

$=\frac{1}{4} *\left[(v(\lambda))^{t}+\left(v\left(\lambda^{t}\right)\right)^{t}\right] *\left[\left(I_{p^{2}}-\left(I_{p} \otimes \Omega\right) \Lambda_{p}\right)(\Omega \otimes \Omega)\left(I_{p^{2}}-\Lambda_{p}\left(I_{p} \otimes \Omega\right)\right)\right] *[(v(\lambda))+$ $\left.\left(v\left(\lambda^{t}\right)\right)\right]$

$=\frac{1}{4} *\left[(v(\lambda))^{t} * I_{p^{2}}+\left(v\left(\lambda^{t}\right)\right)^{t} * I_{p^{2}}-(v(\lambda))^{t} *\left(I_{p} \otimes \Omega\right) \Lambda_{p}-\left(v\left(\lambda^{t}\right)^{t}\right)\left(I_{p} \otimes \Omega\right) \Lambda_{p}\right] *$ $(\Omega \otimes \Omega)\left[\left(I_{p^{2}} v(\lambda)+I_{p^{2}} v\left(\lambda^{t}\right)-\Lambda_{p}\left(I_{p} * \otimes \Omega\right) v(\lambda)-\Lambda_{p}\left(I_{p} \otimes \Omega\right) v\left(\lambda^{t}\right)\right]\right.$

$=\frac{1}{4} *\left[(v(\lambda))^{t}+\left(v\left(\lambda^{t}\right)\right)^{t}-(v(\lambda))^{t} *\left(I_{p} \otimes \Omega\right) \Lambda_{p}-\left(v\left(\lambda^{t}\right)^{t}\right)\left(I_{p} \otimes \Omega\right) \Lambda_{p}\right] *(\Omega \otimes$ $\Omega)\left[v(\lambda)+v\left(\lambda^{t}\right)-\Lambda_{p}\left(I_{p} * \otimes \Omega\right) v(\lambda)-\Lambda_{p}\left(I_{p} \otimes \Omega\right) v\left(\lambda^{t}\right)\right]$

We define $D_{W}$ as a matrix the diagonal elements are the diagonal elements of $W$.

Now

$v(\lambda)^{t} *\left(I_{p} \otimes \Omega\right) \Lambda_{p}=v\left(D_{\Omega \lambda}\right), v\left(\lambda^{t}\right)^{t} *\left(I_{p} \otimes \Omega\right) \Lambda_{p}=v\left(D_{\lambda \Omega}\right)$

$\Lambda_{p} *\left(I_{p} \otimes \Omega\right) * v(\lambda)=v\left(D_{\Omega \lambda}\right), \Lambda_{p} *\left(I_{p} \otimes \Omega\right) * v\left(\lambda^{t}\right)=v\left(D_{\lambda \Omega}\right)$

$K_{m n} * v(A)=v\left(A^{t}\right)$ Theorem 7.30 (Schott, 1997).

Thus, $(v(\lambda))^{t} * K_{p p}=\left(v\left(\lambda^{t}\right)\right)^{t}$

$=\frac{1}{4}\left[v\left(\lambda^{t}\right)(\Omega \otimes \Omega)+\left(v\left(\lambda^{t}\right)^{t}\right)(\Omega \otimes \Omega)-v\left(D_{\Omega \lambda}\right)^{t}(\Omega \otimes \Omega)-v\left(D_{\lambda \Omega}\right)^{t}(\Omega \otimes \Omega)\right][v(\lambda)+$ $\left.v\left(\lambda^{t}\right)-v\left(D_{\Omega \lambda}\right)-v\left(D_{\lambda \Omega}\right)\right]$

$=\frac{1}{4}\left[v\left(\lambda^{t}\right)(\Omega \otimes \Omega) v(\lambda)+\left(v\left(\lambda^{t}\right)^{t}\right)(\Omega \otimes \Omega) v(\lambda)-v\left(D_{\Omega \lambda}\right)^{t}(\Omega \otimes \Omega) v(\lambda)-v\left(D_{\lambda \Omega}\right)^{t}(\Omega \otimes\right.$ $\Omega) v(\lambda)+v\left(\lambda^{t}\right)(\Omega \otimes \Omega) v\left(\lambda^{t}\right)+\left(v\left(\lambda^{t}\right)^{t}\right)(\Omega \otimes \Omega) v\left(\lambda^{t}\right)-v\left(D_{\Omega \lambda}\right)^{t}(\Omega \otimes \Omega) v\left(\lambda^{t}\right)-$ $v\left(D_{\lambda \Omega}\right)^{t}(\Omega \otimes \Omega) v\left(\lambda^{t}\right)-v\left(\lambda^{t}\right)(\Omega \otimes \Omega) v\left(D_{\Omega \lambda}\right)-\left(v\left(\lambda^{t}\right)^{t}\right)(\Omega \otimes \Omega) v\left(D_{\Omega \lambda}\right)+v\left(D_{\Omega \lambda}\right)^{t}(\Omega \otimes$ $\Omega) v\left(D_{\Omega \lambda}\right)+v\left(D_{\lambda \Omega}\right)^{t}(\Omega \otimes \Omega) v\left(D_{\Omega \lambda}\right)-v(\lambda)^{t}(\Omega \otimes \Omega) v\left(D_{\lambda \Omega}\right)-v\left(\lambda^{t}\right)^{t}(\Omega \otimes \Omega) v\left(D_{\lambda \Omega}\right)+$ 
$\left.v\left(D_{\Omega \lambda}\right)^{t}(\Omega \otimes \Omega) v\left(D_{\lambda \Omega}\right)+v\left(D_{\lambda \Omega}\right)^{t}(\Omega \otimes \Omega) v\left(D_{\lambda \Omega}\right)\right]$

Note

$v(\lambda)^{t}(\Omega \otimes \Omega) v(\lambda)=v\left(\lambda^{t}\right)^{t}(\Omega \otimes \Omega) v\left(\lambda^{t}\right), v\left(\lambda^{t}\right)^{t}(\Omega \otimes \Omega) v(\lambda)=v(\lambda)^{t}(\Omega \otimes \Omega) v\left(\lambda^{t}\right)$ $v\left(D_{\Omega \lambda}\right)(\Omega \otimes \Omega) v(\lambda)=v\left(D_{\Omega \lambda}\right)^{t}(\Omega \otimes \Omega) v(\lambda)^{t}=v(\lambda)^{t}(\Omega \otimes \Omega) v\left(D_{\Omega \lambda}\right)=v\left(\lambda^{t}\right)(\Omega \otimes$ $\Omega) v\left(D_{\Omega \lambda}\right), v\left(D_{\lambda \Omega}\right)^{t}(\Omega \otimes \Omega) v\left(D_{\Omega \lambda}\right)=v\left(D_{\Omega \lambda}\right)^{t}(\Omega \otimes \Omega) v\left(D_{\lambda \Omega}\right)$

Now by using Theorem 7.15, 7.16 and 7.17 from [15] and Proposition 1.3.14. from [10] we have the following

$=\frac{1}{4} *\left[2 * \operatorname{trace}\left(\lambda^{t} \Omega \lambda \Omega\right)+2 * \operatorname{trace}\left((\lambda \Omega)^{2}\right)-4 * \operatorname{trace}\left(\lambda^{t} \Omega D_{\lambda \Omega} \Omega\right)-4 * \operatorname{trace}\left(\lambda \Omega D_{\Omega \lambda} \Omega\right)+\right.$ $\left.2 * \operatorname{tarce}\left(D_{\Omega \lambda} \Omega D_{\lambda \Omega} \Omega\right)+\operatorname{trace}\left(\left(D_{\Omega \lambda} \Omega\right)^{2}\right)+\operatorname{trace}\left(\left(D_{\lambda \Omega} \Omega\right)^{2}\right)\right]$

$\sigma^{2}=8 * \frac{1}{4} *\left[2 * \operatorname{trace}\left(\lambda^{t} \Omega \lambda \Omega\right)+2 * \operatorname{trace}\left((\lambda \Omega)^{2}\right)-4 * \operatorname{trace}\left(\lambda^{t} \Omega D_{\lambda \Omega} \Omega\right)-4 *\right.$ $\left.\operatorname{trace}\left(\lambda \Omega D_{\Omega \lambda} \Omega\right)+2 * \operatorname{tarce}\left(D_{\Omega \lambda} \Omega D_{\lambda \Omega} \Omega\right)+\operatorname{trace}\left(\left(D_{\Omega \lambda} \Omega\right)^{2}\right)+\operatorname{trace}\left(\left(D_{\lambda \Omega} \Omega\right)^{2}\right)\right]$

$\sigma^{2}=2 *\left[2 * \operatorname{trace}\left(\lambda^{t} \Omega \lambda \Omega\right)+2 * \operatorname{trace}\left((\lambda \Omega)^{2}\right)-4 * \operatorname{trace}\left(\lambda^{t} \Omega D_{\lambda \Omega} \Omega\right)-4 *\right.$ $\left.\operatorname{trace}\left(\lambda \Omega D_{\Omega \lambda} \Omega\right)+2 * \operatorname{tarce}\left(D_{\Omega \lambda} \Omega D_{\lambda \Omega} \Omega\right)+\operatorname{trace}\left(\left(D_{\Omega \lambda} \Omega\right)^{2}\right)+\operatorname{trace}\left(\left(D_{\lambda \Omega} \Omega\right)^{2}\right)\right]$

\section{Proposed Test}

As we mentioned above the limitations of those tests which involves the determinant of the sample matrix, i.e., generalized variance $(\mathrm{GV})$ as a measure of multivariate dispersion measurement. Due to the application of this measure these test are quite cumbersome when the data in high dimension. Our proposed test used vector variance as a measure of multivariate dispersion, which is equal to a sum square of the elements of the sample correlation.

According to [13] by using multivariate statistical process control (MSPC), the hypothesis testing for the stability of correlation structure $H_{0}: \Omega_{1}=\Omega_{2}=$ $\ldots=\Omega_{m}$ versus $H_{1}: \Omega_{i} \neq \Omega_{j}$ for at least one pair $(i, j)$ is equivalently to repeated the test $H_{0}: \Omega_{i}=\Omega_{0}$ versus $H_{1}: \Omega_{i} \neq_{0}$ where $i=1,2, m$. Where $\Omega_{0}$ is reference sample. The proposed statistical test is

$$
Z_{i}^{*}=\frac{\left\|v\left(R_{i U}\right)\right\|^{2}-E\left(\left\|v\left(R_{U}\right)\right\|^{2}\right)}{\sqrt{\left(\frac{1}{n-1}\right) \sigma^{2}}}=\frac{\left\|v\left(R_{i U}\right)\right\|^{2}-\left(\left\|v\left(\Omega_{U}\right)\right\|^{2}\right)}{\sqrt{\left(\frac{1}{n-1}\right) \sigma^{2}}} .
$$

The null hypothesis $H_{0}$ is rejected at the significance level $\alpha$ when $\mid Z_{i}>z_{\frac{\alpha}{2}}$ with $(1-\alpha / 2)^{t} h$ quantile of standard normal distribution. However, in the case 
of $\Omega$ unknown, it must be estimated from independent random sample $\hat{\Omega}$ where $\hat{\Omega}=\bar{R}$ the average of correlation matrices of $R_{1}, R_{2}, R_{m}$.

\section{Conclusion}

In this paper, the asymptotic distribution of correlation matrices was derived. The distribution can be approximated by standard normal distribution. By using the upper-off-diagonal elements of the matrix to handle the case when $p$ is large.

\section{Acknowledgments}

The authors gratefully acknowledge sponsorship University Utara Malaysia. They also thank the reviewers for their helpful comments and suggestions.

\section{References}

[1] T.W. Anderson, An Introduction to Multivariate Statistical Analysis, Wiley, USA, 2003.

[2] G.E. Box, A general distribution theory for a class of likelihood criteria, Biometrika, 36, No-s: 3, 4 (1949, 317-346.

[3] M. Browne, A. Shapiro, The asymptotic covariance matrix of sample correlation coefficients under general conditions, Linear Algebra and its Applications, 82 (1986), 169-176.

[4] D.C. Cho, W.M. Taylor, The seasonal stability of the factor structure of stock, The Journal of Finance, 42, No. 5 (1987), 1195-1211.

[5] Da N. Costa Jr, S. Nunes, P. Ceretta, S. Da Silva, Stock market comovements revisited, Economics Bulletin, 7, No. 3 (2005), 1-9.

[6] De M.J. Ceuster, J. Annaert, A.G. Claes, Inter-temporal Stability of the European Credit Spread Co-movement Structure, 2003.

[7] M. Djauhari, E. Herdiani, Monitoring the stability of correlation structure in drive rib production process: An MSPC Approach, Open Industrial and Manufacturing Engineering Journal, 1 (2008), 8-18.

[8] R.I. Jennrich, An asymptotic $\chi^{2}$ test for the equality of two correlation matrices, Open Industrial and Manufacturing Engineering Journal, 1 (1970), 8-18.

[9] E.C. Kaplanis, Stability and forecasting of the comovement measures of international stock market returns, Journal of International Money and Finance, 7, No. 1 (1988), 63-75.

[10] T. Kollo, D. von Rosen, Advanced multivariate statistics with matrices, Springer Science and Business Media, 7, No. 1 (2006), 63-75. 
[11] S. Lee, The Inter-Temporal Stability of Real Estate Returns: An empirical investigation, 1998.

[12] I. Meric, G. Meric, Co-movements of European equity markets before and after the 1987 crash, Multinational Finance Journal, 1, No. 2 (1997), 137-152.

[13] D.C. Montgomery, Introduction to Statistical Quality Control, JSTOR, 2005.

[14] H. Neudecker, A.M. Wesselman, The asymptotic variance matrix of the sample correlation matrix, Linear Algebra and its Applications, 127 (1990), 589-599.

[15] J.R. Schott, Matrix Analysis for Statistics, John Wiley and Sons, 1997.

[16] S. Sharif, M.A. Djauhari, Asymptotic derivation of T* statistic, In: Paper Presented at International Conference on Quantitative Sciences and Its Applications (ICOQSIA 2014): Proceedings of the 3-rd International Conference on Quantitative Sciences and Its Applications, 2014.

[17] G.Y. Tang, Stability of international stock market relationships across month of the year and different holding intervals, The European Journal of Finance, 1, No. 3 (1995), 207-218.

[18] G.Y. Tang, The intertemporal stability of the covariance and correlation matrices of Hong Kong stock returns, Applied Financial Economics, 8, No. 4 (1998), 359-365. 